\title{
Two methods for solving electrostatic problems with azimuthal symmetry
}

\author{
T. E. P. Bueno ${ }^{1}$, U. Camara da Silva*1@ \\ ${ }^{1}$ Universidade Federal do Espírito Santo, Departamento de Física, Vitória, ES, Brasil
}

Received on August 23, 2019. Revised on October 2, 2019. Accepted on October 4, 2019.

\begin{abstract}
The study of electrostatic phenomena is the gateway to the physics described by Classical Electrodynamics. In this paper, we discuss in detail two methods based on the Uniqueness Theorem for solving electrostatic problems with azimuthal symmetry. The first one is the electrostatic potential extension from the axis of symmetry to an arbitrary point. The other consists in the mutual mapping between two potentials through an inversion transformation. We have prepared a list of six examples for which we calculate, completely or partially, the electrostatic potentials for different charge distributions using both methods. The electric field lines are analyzed and presented graphically in all cases.
\end{abstract}

Keywords: Electrostatic, Azimuthal Symmetry, Uniqueness Theorem, Method of Inversion.

\section{Introduction}

Classical Electrodynamics (CED), formulated at the end of the nineteenth century, is one of the greatest triumphs of science. It not only unified the already known electric and magnetic phenomena but also predicted the existence of electromagnetic waves and, being the first relativistic theory developed, CED served as a foundation for our current understanding of space and time. Together with the gravitational interaction, the classical electromagnetic fields are responsible for all the physics we observe in our macroscopic daily life.

In CED, the evolution of the electromagnetic (e.m.) field - the electric field $\vec{E}(\vec{x}, t)$ and the magnetic field $\vec{B}(\vec{x}, t)$ - is determined when we solve the so-called Maxwell's Equations

$$
\begin{aligned}
& \vec{\nabla} \cdot \vec{E}(\vec{x}, t)=\frac{\rho(\vec{x}, t)}{\varepsilon_{0}}, \quad \vec{\nabla} \times \vec{E}(\vec{x}, t)+\frac{\partial \vec{B}(\vec{x}, t)}{\partial t}=0, \\
& \vec{\nabla} \cdot \vec{B}(\vec{x}, t)=0, \vec{\nabla} \times \vec{B}(\vec{x}, t)-\mu_{0} \varepsilon_{0} \frac{\partial \vec{E}(\vec{x}, t)}{\partial t}=\mu_{0} \vec{j}(\vec{x}, t),
\end{aligned}
$$

assuming that we already know the dynamics of the electric charges (sources of the e.m. field) described by the densities of charge, $\rho(\vec{x}, t)$, and current, $\vec{j}(\vec{x}, t)$. At the same time, an electromagnetic field defined in space creates a Lorentz force on each charge $q_{i}$ given by

$$
\vec{F}_{i}^{(e . m .)}=q_{i}\left(\vec{E}(\vec{x}, t)+\frac{d \vec{x}_{i}}{d t} \times \vec{B}(\vec{x}, t)\right),
$$

where we assume a set of point charges, i.e. $\rho(\vec{x}, t)=$ $\sum_{i} q_{i} \delta\left(\vec{x}-\vec{x}_{i}(t)\right)$ and $\vec{j}(\vec{x})=\sum_{i} q_{i} \frac{d \vec{x}_{i}(t)}{d t} \delta\left(\vec{x}-\vec{x}_{i}(t)\right)$.

*Correspondence email address: ulyssescamara@gmail.com
Therefore it is not difficult to see that the description of a system formed by electric charges and an e.m. field is a difficult task. In general, we have an endless loop: the electric charges create an e.m. field obeying Maxwell's equations that modifies their dynamics according to the Lorentz force, and so on. Only in simple systems, when we have control over the field configuration or of the charge distribution, there are analytical solutions. Fortunately, in macroscopic scales, a class of simple systems becomes very relevant - the electrostatic phenomena. Electrostatics consists in determining the electric field formed by a previously known macroscopic charge distribution, characterized by the charge density, $\rho(x)$, which does not evolve in time. Textbooks of Basic Physics [1 3 and Classical Electromagnetic Field Theory 4,5. usually dedicate a substantial part of their text to the analysis of electrostatic physics. This article is devoted to the introduction and implementation of two powerful techniques described subtly in the references [6,7]. These methods are little explored in undergraduate courses and allow for the resolution (sometimes only in a partial way) of a wide range of electrostatic problems with azimuthal symmetry. The first technique consists in the determination of the electrostatic potential by an explicit calculation done only on the axis of symmetry. The second technique is the inversion method, in which the potential on the outside of a sphere of radius $R$ is mapped to the inside of it and vice versa, maintaining the boundary conditions on the sphere surface intact.

In section 2, we have a review of the principal properties of the Poisson and Laplace equations that govern the electrostatic phenomena. In section 3 the two methods are derived using the Uniqueness Theorem in the context of problems with azimuthal symmetry. Section 4 provides 
a series of applications that illustrate the advantages and limitations of the two methods. The solved examples are the charged ring, the ring outside/inside of a grounded conducting sphere, the charged hemisphere, the disc, and the rod. Finally, we present our final considerations in section 5

\section{Poisson and Laplace equations}

In an electrostatic situation, $\rho=\rho(\vec{x})$ and $\vec{j}=\overrightarrow{0}$, and equation (2) does not provide any information, since constraint forces compensate the electromagnetic force in such a way that the charges do not move. In the absence of dynamics, there is no magnetic field and Maxwell's Eqs. (1) become only

$$
\vec{\nabla} \cdot \vec{E}(\vec{x})=\frac{\rho(\vec{x})}{\varepsilon_{0}}, \quad \vec{\nabla} \times \vec{E}(\vec{x})=0 .
$$

The second equation implies that the electric field is conservative. So it can be rewritten in terms of the scalar potential, $\vec{E}(\vec{x})=-\vec{\nabla} \phi(\vec{x})$. Substituting this new form into the first equation of $(3)$ we have the following result

$$
\nabla^{2} \phi(\vec{x})=-\frac{\rho(\vec{x})}{\varepsilon_{0}}
$$

the so-called Poisson's Equation which describes all electrostatic physics and have very particular characteristics. The most important one is the Uniqueness Theorem: it says that if Dirichlet or Neumann boundary conditions are given, respectively

$$
\left.\phi\right|_{\partial V} \quad \text { or }\left.\quad \hat{n} \cdot \vec{\nabla} \phi\right|_{\partial V},
$$

where $\partial V$ is a closed surface that encloses the volume $V$ and $\hat{n}$ is the unit vector normal to the surface, then the potential $\phi(x)$ is unique in all points of interest. The proof of the theorem can found in several books on the subject [4 6]. In this article, we will deal only with Dirichlet boundary conditions.

For a localized charge distribution, i.e. when all charges are inside of a sphere of finite radius, we must impose Dirichlet boundary condition, $\left.\phi\right|_{\partial \mathbb{R}^{3}}=0$, and $\phi(\vec{x})$ is determined (by the Uniqueness Theorem) as 4

$$
\phi(\vec{x})=\frac{1}{4 \pi \varepsilon_{0}} \int_{\mathbb{R}^{3}} \frac{\rho\left(\vec{x}^{\prime}\right)}{\left|\vec{x}-\vec{x}^{\prime}\right|} d^{3} x^{\prime} .
$$

The solution given by equation (6) has a problem at the practical level: even for simple charge configurations, it can lead to complicated integrals. We will discuss this point in section 3.1 .

In points of space without charges, we have Laplace's equation,

$$
\nabla^{2} \phi(\vec{x})=0
$$

An important fact is that the scalar potential has no minimum or maximum at the points where equation
(7) is valid - Earnshaw's Theorem. As a consequence there is no distribution of static charges resulting in a stable configuration, i.e. binding forces are required to maintain an electrostatic situation. One must take some caution in the theorem's proof, it is not uncommon the use of conceptually wrong arguments in this task, see reference [8] for a comprehensible discussion about the subject. A correct and elegant demonstration is found on page 3 of $[7]$. It consists of assuming that $\phi(\vec{x})$, solution of (7), has a minimum (maximum) at point $\vec{x}_{0}$. If this is true, necessarily around the point $\vec{x}_{0}$ there is an arbitrarily small closed surface $\partial V$, the border of volume $V$, where the flux of the vector field $\vec{\nabla} \phi$ is positive (negative). Therefore

$$
\oint_{\partial V} \vec{\nabla} \phi \cdot \hat{n} d^{2} x>0 \quad\left(\oint_{\partial V} \vec{\nabla} \phi \cdot \hat{n} d^{2} x<0\right) .
$$

On the other hand, using Gauss's theorem 9]

$$
\oint_{\partial V} \vec{\nabla} \phi \cdot \hat{n} d^{2} x=\int_{V} \nabla^{2} \phi d^{3} x \stackrel{E q \cdot \sqrt{7}}{=} 0,
$$

then a point of minimum (maximum) is not compatible with the Laplace Equation and the theorem is proved by contradiction.

\section{Methods for solving problems with azimuthal symmetry}

We are interested in electrostatic situations with azimuthal symmetry. In this case, using spherical coordinates, $(r, \theta, \varphi)$, the electric potential is not a function of the azimuthal angle $\varphi \in[0,2 \pi]$. The general solution of equation (7) for this symmetry with $\theta \in[0, \pi]$ is given by 4,5

$$
\phi(r, \theta)=\sum_{l=0}^{\infty}\left(A_{l} r^{l}+\frac{B_{l}}{r^{l+1}}\right) P_{l}(\cos \theta),
$$

where $P_{l}(\cos \theta)$ are the Legendre Polynomials, in the appendix A, we list some of their properties. Then, in a region without charges, every electrostatic problem with azimuthal symmetry consists in the search of the set of parameters $\left\{A_{l}\right.$ e $\left.B_{l}, l=0,1, \ldots\right\}$. In the rest of the section, we shall introduce two methods that can help in this task.

\subsection{Extension around the axis of symmetry}

Assume a localized distribution of electric charges which has azimuthal symmetry, and in which there are no charges inside the volume determined by $r_{\min }<r<$ $r_{\max }$. In this region, the potential is given simultaneously by Eqs. (6) and (8). Let us assume that the integral (6) is difficult to solve at an arbitrary point, but it is simple along the $z$-axis of symmetry. A ring, a disk and a rod with uniform distributions of charges are examples of this type of situation, and we will explore them in the 
next section. Performing the integration only on the axis of symmetry, we obtain the exact result

$$
\begin{aligned}
& \phi(z)=\frac{1}{4 \pi \varepsilon_{0}} \int_{\mathbb{R}^{3}} \frac{\rho\left(\vec{x}^{\prime}\right)}{z \hat{z}-\vec{x}^{\prime} \mid} d^{3} x^{\prime}=\mathcal{F}(z), \\
& r_{\text {min }}<|z|<r_{\text {max }} .
\end{aligned}
$$

On the other hand, the same solution can be written in terms of the series (8), the positive half-axis $(z>0)$ being given by $r=|z|=z$ and $\theta=0$ and the negative half-axis $(z<0)$ given by $r=|z|=-z$ and $\theta=\pi$. For these points, the Legendre Polynomials assume the values, see equation 2 (Appendix A)

$$
P_{l}(\cos \theta)= \begin{cases}1 & \theta=0 \\ (-1)^{l} & \theta=\pi\end{cases}
$$

Taking this into account, the function $\mathcal{F}(z)$ can be rewritten as

$\mathcal{F}( \pm|z|)=\phi( \pm|z|)=\sum_{l=0}^{\infty}( \pm 1)^{l}\left(A_{l}|z|^{l}+\frac{B_{l}}{|z|^{l+1}}\right)$

The expansion of $\mathcal{F}(z)$ in powers series of $|z|$ and/or $1 /|z|$ completely determine the coefficients $A_{l}$ and $B_{l}$. So, by the Uniqueness Theorem, the scalar potential is fixed in a unique way for all theta $\theta \in[0, \pi]$ and $r_{\min }<r<r_{\max }$, see equation (8).

\subsection{Method of Inversion}

Again we assume that there are no charges in the region $r_{\min }<r<r_{\max }$ and the electrostatic potential is given by equation (8). Now for some $R$ such that $r_{\text {min }}<R<$ $r_{\max }$ we define the function

$$
\bar{\phi}(r, \theta) \equiv \frac{R}{r} \phi\left(\frac{R^{2}}{r}, \theta\right),
$$

so

$$
\begin{aligned}
\bar{\phi}(r, \theta) & =\frac{R}{r} \sum_{l=0}^{\infty}\left(A_{l} \frac{R^{2 l}}{r^{l}}+\frac{B_{l}}{R^{2 l+2}} r^{l+1}\right) P_{l}(\cos \theta) \\
& =\sum_{l=0}^{\infty}\left(\frac{B_{l}}{R^{2 l+1}} r^{l}+A_{l} \frac{R^{2 l+1}}{r^{l+1}}\right) P_{l}(\cos \theta) \\
& =\sum_{l=0}^{\infty}\left(\bar{A}_{l} r^{l}+\frac{\bar{B}_{l}}{r^{l+1}}\right) P_{l}(\cos \theta)
\end{aligned}
$$

where $\bar{A}_{l}=B_{l} / R^{l}$ and $\bar{B}_{l}=A_{l} R^{2 l+1}$. This function is a solution of the Laplace's Equation, thus the original potential $\phi(r, \theta)$, with $r_{\text {min }}<r<r_{\max }$, was mapped to another electrostatic potential $\bar{\phi}(r, \theta)$ which is now defined in the interval $R^{2} / r_{\max }<r<R^{2} / r_{\min }$. This procedure can always be done allowing, a priori, the construction of two potentials for the price of one. An interesting point to note is that by performing this operation twice, we return to the original potential, i.e. $\overline{\bar{\phi}}(r, \theta)=\phi(r, \theta)$.
The main question here is when the Method of Inversion is useful. This occurs when we have boundary conditions exactly on the sphere of radius $R$. Note that by construction we have $\bar{\phi}(R, \theta)=\phi(R, \theta)$, therefore the operation maps the part of the potential $\phi$ defined outside/inside the sphere at the interior/exterior region of the potential $\bar{\phi}$ and vice-versa. Both obey the same boundary condition at $r=R$. This situation arises when we have a distribution of charges on the spherical shell of radius $R$ with boundary condition $\phi(R, \theta)=V(\theta)$.

In this case, if $\phi(r, \theta)$ describes the outside region $r>R$ (so we must have $A_{l}=0$ for the potential to vanish as $r \rightarrow \infty)$, then, automatically, the function $\bar{\phi}(r, \theta)$, with $\bar{A}_{l}=B_{l} / R^{l}$, is the potential in the interior region, $r<R$ (now we have $\bar{B}_{l}=A_{l} R^{2 l+1}=0$ for finitude at the origin). A second possibility is the mapping between two different physical situations: one problem is defined in $R<r<r_{\max }$ and the other in $R^{2} / r_{\max }<r<R$, including the limit $r_{\max } \rightarrow \infty$, both having the same boundary condition $\phi(R, \theta)=V(\theta)$.

\section{Applications}

Here we will use the methods developed in the previous section to obtain the electrostatic potential in terms of power series for several examples. The problems were chosen to illustrate the advantages and limitations of this approach. With the electrostatic potential already known, the electric field is determined by direct calculation of $\vec{E}=-\vec{\nabla} \phi(r, \theta)$. In this way, we will describe in all cases the electric field graphically using the Wolfram Mathematica software. Explicitly, the figures were made using the command StreamPlot of the software and represent the field lines in the plane $x=0$.

\subsection{Ring of charge}

Consider a thin ring of radius $R$ and uniform charge density $\lambda$ (total charge $q=2 \pi R \lambda$ ), see figure 1 . The calculation of the potential at an arbitrary point using equation (6) is not a trivial task. On the other hand, the integral only on the $z$-axis is a simple exercise illustrated in several basic physics books. The result is [1]

$$
\phi(z)=\frac{q}{4 \pi \varepsilon_{0}} \frac{1}{\sqrt{z^{2}+R^{2}}} .
$$

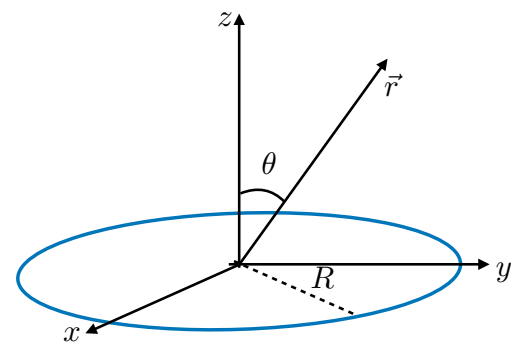

Figure 1: Ring with uniform charge density. 
This exercise is the standard one to illustrate the method of extension around the axis of symmetry 6]. To extend this solution we must expand equation (14) in power series. There are two distinct expansions, one in the region $|z|>R$ and another in $|z|<R$. Let us start with $z>R$, so equation (14) can be rewritten as

$$
\begin{aligned}
\phi(z) & =\frac{q}{4 \pi \varepsilon_{0}} \frac{1}{\sqrt{z^{2}+R^{2}}}=\frac{q}{4 \pi \varepsilon_{0}} \frac{1}{z} \frac{1}{\sqrt{1+\frac{R^{2}}{z^{2}}}}, \\
& =\frac{q}{4 \pi \varepsilon_{0}} \sum_{n=0}^{\infty} \frac{(-1)^{n}}{2^{2 n}} \frac{(2 n) !}{(n !)^{2}} \frac{R^{2 n}}{z^{2 n+1}}
\end{aligned}
$$

where equation 4 (Appendix A) was used. Comparing with equation (11) we have that $A_{l}=0$ and only the even $B_{l}$ 's are non-zero, i.e. $l=2 n, n \in \mathbb{N}$. Thus, as described in section 3.1 the potential outside the sphere of radius $R$ is defined uniquely by exchanging $z$ for $r$ and inserting the corresponding Legendre Polynomials. The complete solution is

$$
\begin{array}{r}
\phi_{>}^{r i n g}(r, \theta)=\frac{q}{4 \pi \varepsilon_{0} R} \sum_{n=0}^{\infty} \frac{(-1)^{n}}{2^{2 n}} \frac{(2 n) !}{(n !)^{2}}\left(\frac{R}{r}\right)^{2 n+1} \\
\times P_{2 n}(\cos \theta),
\end{array}
$$

$r \geq R$. In the inner region, $|z|<R$, again using the equation 4 (Appendix A) we have the expansion $(z>0)$

$$
\begin{aligned}
\phi(z) & =\frac{q}{4 \pi \varepsilon_{0}} \frac{1}{\sqrt{z^{2}+R^{2}}}=\frac{q}{4 \pi \varepsilon_{0} R} \frac{1}{\sqrt{1+\frac{z^{2}}{R^{2}}}} \\
& =\frac{q}{4 \pi \varepsilon_{0} R} \sum_{n=0}^{\infty} \frac{(-1)^{n}}{2^{2 n}} \frac{(2 n) !}{(n !)^{2}} \frac{z^{2 n}}{R^{2 n}} .
\end{aligned}
$$

Now the terms $B_{l}$ are all null and only the even parameters $A_{l}$ 's are non-zero, i.e. $l=2 n, n \in \mathbb{N}$. The potential inside the sphere of radius $R$ is

$$
\begin{aligned}
\phi_{<}^{r i n g}(r, \theta)=\frac{q}{4 \pi \varepsilon_{0} R} \sum_{n=0}^{\infty} \frac{(-1)^{n}}{2^{2 l}} & \frac{(2 n) !}{(n !)^{2}}\left(\frac{r}{R}\right)^{2 n} \\
& \times P_{2 n}(\cos \theta),
\end{aligned}
$$

$0 \leq r \leq R$. The two solutions coincide in $r=R$, guaranteeing the continuity of potential. Continuity is a necessary condition, but the intersection of the two solutions being a sphere is a curious fact, since the problem is about a ring of charge. This is a consequence of the azimuthal symmetry. It is important to note that the calculation of the two solutions in a separate way was not necessary. One solution determines the other by the inversion method. It is straightforward to verify $\phi_{<}^{r i n g}(r, \theta)=(R / r) \phi_{>}^{r i n g}\left(R^{2} / r, \theta\right)$. The electric field lines are illustrated in figure 2 , showing the plane $\{x=0\}$, i.e. a "lateral cut" of the ring geometry (the ring is on the plane $\{z=0\})$. As one can see, in the inside of the spherical region of radius $R$ the field lines move away from the plane of the ring; far outside of the ring, the lines approach a radial behavior, which is the expected result for a point charge.

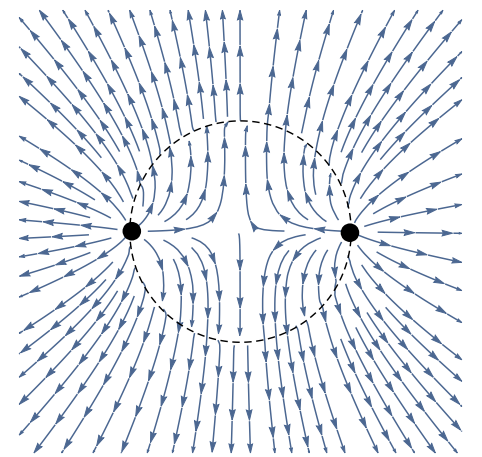

Figure 2: Electric field lines on the plane $x=0$ for case 4.1 . The two points represent the location of the ring on the plane; the dashed circle indicates the sphere of radius $R$.

\subsection{Charged ring outside a conducting sphere}

Our second example is "Saturn's ring". We want to calculate the electric field generated by a grounded conducting sphere of radius $R$ and a charged ring of radius $b>R$ and with uniform charge density $\lambda$ (total charge $q=2 \pi b \lambda$ ), in the entire region outside of the sphere, where $r>R$. See figure $3 a$

Our approach will be via the method of images. The presence of the charged ring induces opposite charges on the surface of the grounded conductor in such a way that $\phi(R, \theta)=0$. So we propose to replace the sphere by an image ring of radius $b_{I}<R$ and uniform density $\lambda_{I}$ (total charge $q_{I}=2 \pi b_{I} \lambda_{I}$ ) in such a way that the physical condition $\phi(R, \theta)=0$ is preserved for all $\theta \in[0, \pi]$, see figure $3 \mathrm{~b}$. The Uniqueness Theorem guarantees that if such a configuration exists, it creates the same potential as the original problem. Using Eqs. (16) and (17) our potential ansatz is

$$
\phi(r, \theta)= \begin{cases}\phi_{<}^{\text {ring }}(r, \theta)+\phi_{>}^{\text {image }}(r, \theta), & R \leq r \leq b, \\ \phi_{>}^{\text {ring }}(r, \theta)+\phi_{>}^{\text {image }}(r, \theta), & r \geq b .\end{cases}
$$

At the surface $r=R$ the potential must be null, so

$$
\begin{aligned}
& \phi_{<}^{\text {ring }}(R, \theta)=-\phi_{>}^{\text {image }}(R, \theta), \\
& \frac{q}{4 \pi \varepsilon_{0} b} \sum_{n=0}^{\infty} \frac{(-1)^{n}}{2^{2 n}} \frac{(2 n) !}{(n !)^{2}}\left(\frac{R}{b}\right)^{2 n} P_{2 n}(\cos \theta)= \\
& \frac{q_{I}}{4 \pi \varepsilon_{0} b_{I}} \sum_{n=0}^{\infty} \frac{(-1)^{n}}{2^{2 n}} \frac{(2 n) !}{(n !)^{2}}\left(\frac{b_{I}}{R}\right)^{2 n+1} P_{2 n}(\cos \theta) .
\end{aligned}
$$

The orthogonality of Legendre Polynomials says that this equality holds only if

$$
\left(\frac{R}{b}\right)^{2 n} \frac{q}{b}=-\left(\frac{b_{I}}{R}\right)^{2 n} \frac{q_{I}}{R}, \quad \forall n ; n=0,1,2, \ldots
$$

In particular, for $n=0$ we have $q_{I}=-(R / b) q$, hence $b_{I}=R^{2} / b$. The respective choices

$$
q_{I}=-\left(\frac{R}{b}\right) q, \quad b_{I}=\left(\frac{R}{b}\right) R<R
$$




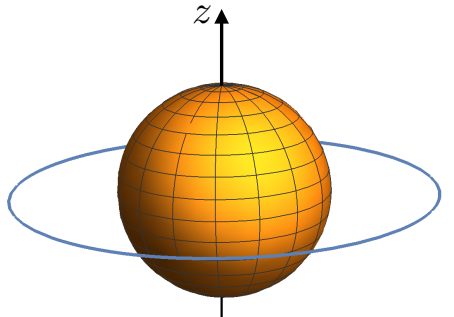

(a)

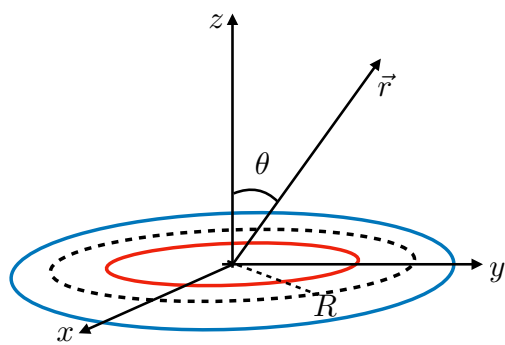

(b)

Figure 3: (a) Grounded conducting sphere of radius $R$ and ring of charge with $b>R$. (b) Equivalent system described by image ring of radius $b_{I}=(R / b) R<R$.

provide the right boundary condition on the conductor surface, which determines the potential. In the region $R \leq r \leq b$ we have

$$
\begin{aligned}
& \phi_{<}(r, \theta)=\frac{q}{4 \pi \varepsilon_{0} b} \sum_{n=0}^{\infty} \frac{(-1)^{n}}{2^{2 n}} \frac{(2 n) !}{(n !)^{2}} \times \\
& {\left[\left(\frac{r}{b}\right)^{2 n}-\left(\frac{R}{b}\right)^{4 n+1}\left(\frac{b}{r}\right)^{2 n+1}\right] P_{2 n}(\cos \theta),}
\end{aligned}
$$

while for $r \geq b$, we have

$$
\begin{aligned}
& \phi_{>}(r, \theta)=\frac{q}{4 \pi \varepsilon_{0} b} \sum_{n=0}^{\infty} \frac{(-1)^{n}}{2^{2 n}} \frac{(2 n) !}{(n !)^{2}} \times \\
& {\left[\left(\frac{b}{r}\right)^{2 n+1}-\left(\frac{R}{b}\right)^{4 n+1}\left(\frac{b}{r}\right)^{2 n+1}\right] P_{2 n}(\cos \theta) .}
\end{aligned}
$$

By direct inspection, it is not difficult to see that the potential is continuous on the sphere of radius $r=b$. The induced charge density on the grounded conducting sphere surface is given by $\sigma(\theta)=\varepsilon_{0} E_{r}(R, \theta)=$ $-\varepsilon_{0} \partial \phi_{<}(R, \theta) / \partial r$, so

$$
\begin{array}{r}
\sigma(\theta)=-\frac{q(R / b)}{4 \pi R^{2}} \sum_{n=0}^{\infty} \frac{(-1)^{n}(4 n+1)(2 n) !}{2^{2 n}(n !)^{2}}\left(\frac{R}{b}\right)^{2 n} \\
\times P_{2 n}(\cos \theta) .
\end{array}
$$

In figure 4 one can see the curve $\left(4 \pi R^{2} / q\right) \sigma(\theta)$ for several values of the ratio $R / b$. For $R / b \ll 1$ (the ring is much larger than the sphere) the induced charge density is approximately uniform and given by the first term of the series, $\sigma(\theta) \approx-q(R / b) /\left(4 \pi R^{2}\right)$. When $R / b \sim 1$ (the

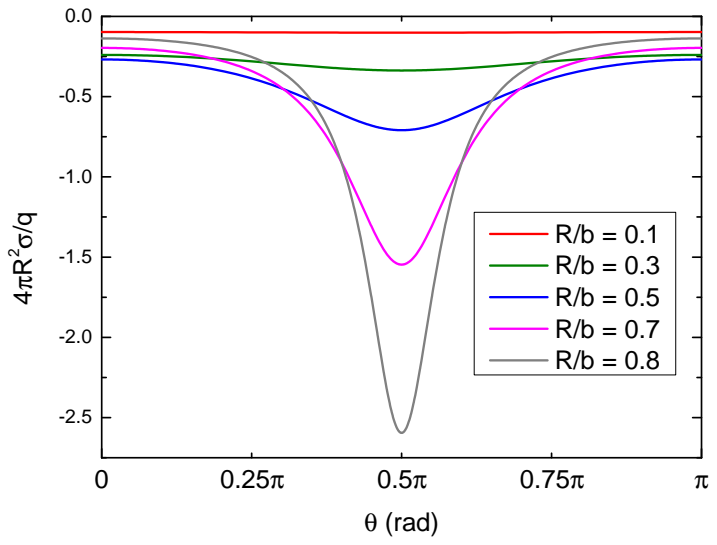

Figure 4: Surface charge density as a function of the polar angle $\theta$. Red $R / b=0.1$, green $R / b=0.3$, blue $R / b=0.5$, purple $R / b=0.7$ and grey $R / b=0.8$.

ring is close to the conducting surface) a concentration of charges appears in the plane of the ring $(\theta=\pi / 2)$. The integral below provides the total induced charge $Q_{\text {ind }}$ on the grounded conductor

$$
\begin{aligned}
& Q_{\text {ind }}=2 \pi R^{2} \int_{-1}^{1} d(\cos \theta) \sigma(\theta) \\
& =-\frac{q(R / b)}{2} \sum_{n=0}^{\infty} \frac{(-1)^{n}(4 n+1)(2 n) !}{2^{2 n}(n !)^{2}}\left(\frac{R}{b}\right)^{2 n} \times \\
& \int_{-1}^{1} d(\cos \theta) P_{2 n}(\cos \theta) \underbrace{P_{0}(\cos \theta)}_{=1}, \\
& =-\frac{q(R / b)}{2} \sum_{n=0}^{\infty} \frac{(-1)^{n}(4 n+1)(2 n) !}{2^{2 n}(n !)^{2}}\left(\frac{R}{b}\right)^{2 n} \\
& \times \frac{2 \delta_{2 n, 0}}{4 n+1},=-\frac{R}{b} q .
\end{aligned}
$$

where equation 6 (Appendix A) was used. As expected, this is the value of the total charge of the image ring. In Figure 5 the electric field lines for $b=2 R$ are shown. An important characteristic is that inside of the fictitious sphere of radius $b$ the radial component of the electric field, i.e. $-\partial \phi / \partial r$, is negative. As a consequence all field lines point inwards and terminate in the conducting surface. The field lines far away from the system have a radial aspect (a point charge behavior).

\subsection{Ring of charge inside a grounded conducting sphere}

This is the "inverse" of the previous problem. A ring of radius $a$ and uniform charge density $\lambda$ (total charge $q=$ $2 \pi a \lambda$ ) is inside a grounded conducting sphere of radius $R$, see figure 6a. We wish to obtain the electric field in the region $0 \leq r \leq R$. This exercise is solved respectively in sections 9.4 and 3.10 of references 5,6 via the spherical Green's function expansion. Here we will directly use the results derived in subsection 4.2 together with the 


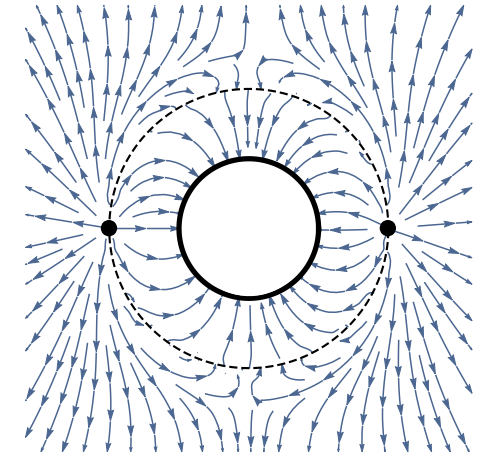

Figure 5: Electric field lines in the plane $\{x=0\}$ for the case 4.2 with $b=2 R$. The two black dots represent the intersection of the ring with the plane; the dashed circle indicates the sphere of radius $b$, and the full black circle is the grounded conductor of radius $R$.

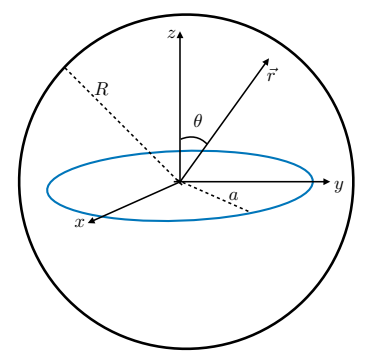

(a)

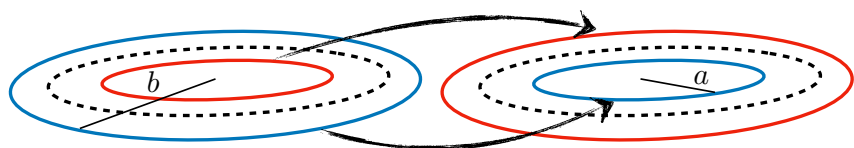

(b)

Figure 6: (a) Ring of charge with radius $a$ inside a grounded conducting sphere of radius $R$. (b) The inversion method maps the real ring (in blue) to the image ring (in red), and vice-versa. The conducting sphere (dashed circle) is not affected by the operation.

method of inversion, $r \rightarrow R^{2} / r$. With the inversion, the intervals $r \geq b$ and $R \leq r \leq b$ are mapped, respectively, to $0 \leq r \leq(R / b) R$, and $(R / b) R \leq r \leq R$. In this procedure, it is clear that the outer and inner rings have changed places, so the physical ring now has radius $a=(R / b) R<R$, while the image ring has radius $b=$ $(R / a) R>R$. That is, the inversion method swapped the real ring and the image ring, keeping the sphere of radius $R$ invariant, see figure $6 \mathrm{~b}$ The same is true to the image charge $q_{I}=-(R / b) q \rightarrow q_{I}=-\left(R a / R^{2}\right) q=-(a / R) q<$ $q$. The final electrostatic potential is

$$
\phi(r, \theta)=\left.\frac{R}{r} \phi_{\text {saturn }}\left(\frac{R^{2}}{r}, \theta\right)\right|_{b=\frac{R^{2}}{a}},
$$

where $\phi_{\text {saturn }}$ is given by Eqs. (20) and (21). In the region $0 \leq r \leq a$, we have

$$
\begin{aligned}
& \phi_{<}(r, \theta)=\frac{q}{4 \pi \varepsilon_{0} R} \sum_{n=0}^{\infty} \frac{(-1)^{n}}{2^{2 n}} \frac{(2 n) !}{(n !)^{2}} \times \\
& {\left[\left(\frac{r}{a}\right)^{2 n}-\left(\frac{a}{R}\right)^{4 n+1}\left(\frac{r}{a}\right)^{2 n}\right] P_{2 n}(\cos \theta) .}
\end{aligned}
$$

while in $a \leq r \leq R$, the potential is

$$
\begin{aligned}
& \phi_{>}(r, \theta)=\frac{q}{4 \pi \varepsilon_{0} R} \sum_{n=0}^{\infty} \frac{(-1)^{n}}{2^{2 n}} \frac{(2 n) !}{(n !)^{2}} \times \\
& {\left[\left(\frac{a}{r}\right)^{2 n+1}-\left(\frac{a}{R}\right)^{4 n+1}\left(\frac{r}{a}\right)^{2 n}\right] P_{2 n}(\cos \theta),}
\end{aligned}
$$

From this result, we extract the surface charge density on the conductor

$$
\begin{aligned}
& \sigma(\theta)=-\varepsilon_{0} \frac{\partial \phi_{>}(R, \theta)}{\partial r} \\
& =-\frac{q(a / R)}{4 \pi \varepsilon_{0} R^{2}} \sum_{n=0}^{\infty} \frac{(-1)^{n}(4 n+1)}{2^{2 n}} \frac{(2 n) !}{(n !)^{2}}\left(\frac{a}{R}\right)^{2 n} \\
& \times P_{2 n}(\cos \theta)
\end{aligned}
$$

which is exactly equation 22 with $R / b \rightarrow a / R$. The curves in figure 4 are also valid here (with the corresponding exchange) and the induced charge is equal to the image charge $Q_{\text {ind }}=-q(a / R)$. The electric field lines for $a=R / 2$ are as described in figure 7. All lines end at the conductor surface, and note that for $r<a$ the lines "run away" from the plane of the ring.

\subsection{Hemisphere with uniform charge density}

The calculation of the electric field generated by a hemisphere (shell only) of radius $R$ and constant charge density $\sigma$ (total charge $q=\sigma\left(2 \pi R^{2}\right)$ ) illustrates a nontrivial application of the two methods described in section 3

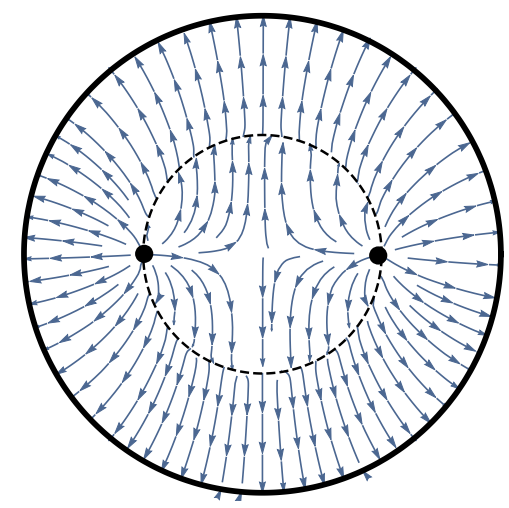

Figure 7: Electric field lines on the plane $\{x=0\}$ for case 4.3 with $a=R / 2$. The two black dots represent the intersection of the ring with the plane, the dashed circle indicates the sphere of radius $a$, and the full black circle is the grounded conductor of radius $R$. 
The first step is to determine the potential in the $z$-axis of symmetry. We can imagine the hemisphere as a "pile of rings", each one with radius $R \sin \theta$, thickness $R d \theta$ and charge $d q=\sigma(2 \pi R \sin \theta)(R d \theta)$, with $\theta \in[0, \pi / 2]$; see figure 8. By the Superposition Principle, the total potential in the z-axis (see equation (14)) is the sum of the potential created by each "ring" so we have

$$
\begin{aligned}
\phi(z) & =\frac{\sigma 2 \pi R^{2}}{4 \pi \varepsilon_{0}} \int_{0}^{\frac{\pi}{2}} \frac{\sin \theta}{\sqrt{(z-R \cos \theta)^{2}+R^{2} \sin ^{2} \theta}} d \theta \\
& =\frac{q}{4 \pi \varepsilon_{0}} \int_{0}^{\frac{\pi}{2}} \frac{\sin \theta}{\sqrt{z^{2}+R^{2}-2 z R \cos \theta}} d \theta, u=\cos \theta \\
& =\frac{q}{4 \pi \varepsilon_{0}} \int_{0}^{1} \frac{1}{\sqrt{z^{2}+R^{2}-2 z R u}} d u \\
& =\left.\frac{q}{4 \pi \varepsilon_{0}} \frac{2}{(-2 z R)} \sqrt{z^{2}+R^{2}-2 z R u}\right|_{u=0} ^{u=1}, \\
& =\frac{q}{4 \pi \varepsilon_{0} R z}\left(\sqrt{z^{2}+R^{2}}-|R-z|\right) \\
& =\frac{q}{4 \pi \varepsilon_{0} z}\left(\sqrt{1+\frac{z^{2}}{R^{2}}}-\left|1-\frac{z}{R}\right|\right) .
\end{aligned}
$$

The existence of the modulus $|1-z / R|$ in the final answer reflects the fact that the parity transformation $z \rightarrow-z$ is not a symmetry in this case. The expansion of equation (28) as a power series of $z$, for $|z|<R$, and of $1 / z$, for $|z|>R$, is sufficient to determine the potential at all points.

Using equation 5 (Appendix A) in the region $z>R$ (let us consider $z>0$ ), the potential can be rewritten as

$$
\begin{aligned}
& \phi(z)=\frac{q}{4 \pi \varepsilon_{0} z}\left(\sqrt{1+\frac{z^{2}}{R^{2}}}-\frac{z}{R}+1\right) \\
& =\frac{q}{4 \pi \varepsilon_{0} R}\left(\sqrt{1+\frac{R^{2}}{z^{2}}}-1+\frac{R}{z}\right) \\
& =\frac{q}{4 \pi \varepsilon_{0}}\left(\frac{1}{z}-\frac{1}{R} \sum_{n=1}^{\infty} \frac{(-1)^{n}}{2^{2 n}(2 n-1)} \frac{(2 n) !}{(n !)^{2}}\left(\frac{R}{z}\right)^{2 n}\right)
\end{aligned}
$$

Note that the zero-term cancels in the sum. equation 29 is sufficient to determine the potential at all points $r>R$. The term separated from the series corresponds to the "point charge" contribution and its value outside the axis

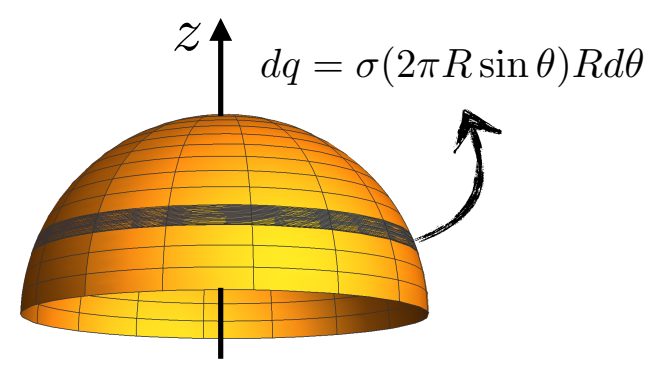

Figure 8: Hemisphere of radius $R$ and uniform charge density. of symmetry is obtained via the formal correspondence $z \rightarrow r$; for $r \gg R$ this term dominates, and effectively we have an electric charge $q$ at the origin. Comparing with equation (11) we have $A_{l}=0, \forall l$, and nonzero $B_{l}$ for $l=0$ and $l=2 n-1, n \in \mathbb{N}^{*}$. By introducing the radial coordinate and the Legendre polynomials, the final form of the potential in this region is

$$
\begin{aligned}
& \phi_{>}(r, \theta)=\frac{q}{4 \pi \varepsilon_{0} r} \\
& -\frac{q}{4 \pi \varepsilon_{0} R} \sum_{n=1}^{\infty} \frac{(-1)^{n}}{2^{2 n}(2 n-1)} \frac{(2 n) !}{(n !)^{2}}\left(\frac{R}{r}\right)^{2 n} \\
& \times P_{2 n-1}(\cos \theta),
\end{aligned}
$$

$r>R$. The results of section 3.1 guarantee that this solution is the electrostatic potential for all $r>R$, including $z<0$. This conclusion is not intuitive since there is no parity symmetry, $z \rightarrow-z$, in this example. To leave no doubts for the reader, we will perform the expansion on the negative half-axis $z<0$ with $|z|>R$. In this case, Eqs. 28 and Appendix A provide

$$
\begin{aligned}
& \phi(-|z|)=-\frac{q}{4 \pi \varepsilon_{0}|z|}\left(\sqrt{1+\frac{|z|^{2}}{R^{2}}}-1-\frac{|z|}{R}\right) \\
& =\frac{q}{4 \pi \varepsilon_{0}}\left(\frac{1}{|z|}+\frac{1}{R} \sum_{n=1}^{\infty} \frac{(-1)^{n}}{2^{2 n}(2 n-1)} \frac{(2 n) !}{(n !)^{2}}\left(\frac{R}{|z|}\right)^{2 n}\right) .
\end{aligned}
$$

As expected the series (28) and (31) are different. On the other hand, in order to extend (31) away from the symmetry axis it is necessary to use the negative sign of equation 11$)\left(P_{l}(-1)=(-1)^{l}\right)$. In this particular problem, only the odd terms are relevant, $l=2 n-1$, $n=1,2, \ldots$, so $P_{2 n-1}(-1)=-1$. Therefore we have to take $|z| \rightarrow r$ and $-1 \rightarrow P_{2 n-1}(\cos \theta)$. This change of sign restores the negative sign in the second term, and following the previous steps we have equation $(30)$ again.

In the region $r \leq R$ the expansion of equation (28) must be done in powers of $z$. Assuming $z$ positive and again with the help of equation 5 (Appendix A) we have

$$
\begin{aligned}
& \phi(z)=\frac{q}{4 \pi \varepsilon_{0} z}\left(\sqrt{1+\frac{z^{2}}{R^{2}}}-\left|1-\frac{z}{R}\right|\right) \\
& =\frac{q}{4 \pi \varepsilon_{0} z}\left(\sqrt{1+\frac{z^{2}}{R^{2}}}-1+\frac{z}{R}\right) \\
& =\frac{q}{4 \pi \varepsilon_{0} R}\left(1-\sum_{n=1}^{\infty} \frac{(-1)^{n}}{2^{2 n}(2 n-1)} \frac{(2 n) !}{(n !)^{2}}\left(\frac{z}{R}\right)^{2 n-1}\right) .
\end{aligned}
$$

In comparison with equation (11) we have $B_{l}=0, \forall l$, and $A_{l} \neq 0$ for $l=0$ and $l=2 n-1, n \in \mathbb{N}^{*}$. Introducing the Legendre polynomials we have the solution

$$
\begin{array}{r}
\phi_{<}(r, \theta)=\frac{q}{4 \pi \varepsilon_{0} R}\left(1-\sum_{n=1}^{\infty} \frac{(-1)^{n}}{2^{2 n}(2 n-1)} \frac{(2 n) !}{(n !)^{2}} \times\right. \\
\left.\left(\frac{r}{R}\right)^{2 n-1} P_{2 n-1}(\cos \theta)\right),
\end{array}
$$


valid for all points $r<R$. Through direct calculation, it is possible to see that the expansion in the negative semiaxis provides the same result. The potential is continuous (as it should be) along the sphere of radius $R$,

$$
\begin{aligned}
& \phi_{<}(R, \theta)=\phi_{>}(R, \theta) \\
& =\frac{q}{4 \pi \varepsilon_{0} R}\left(1-\sum_{n=1}^{\infty} \frac{(-1)^{n}}{2^{2 n}(2 n-1)} \frac{(2 n) !}{(n !)^{2}} P_{2 n-1}(\cos \theta)\right) .
\end{aligned}
$$

We can interpret this result as a boundary condition on the closed surface $r=R$. As a consequence, the regions $r<R$ and $r>R$ are connected by the inversion method. It is easy to verify that

$$
\phi_{>}(r, \theta)=\frac{R}{r} \phi_{<}\left(\frac{R^{2}}{r}, \theta\right) .
$$

The electric field lines are not symmetric about the $z=0$ plane, see figure 9 Far from the system, the electric field becomes radial, such as that of a point charge.

One last comment comes from observing how the electrostatic potential would be if the concavity of the hemisphere were upward. To obtain this potential, we do a parity transformation $z \rightarrow-z$ to our final results. In spherical coordinates, parity becomes $r \rightarrow r$ and $\theta \rightarrow \pi-\theta$. All dependence in the polar angle is inside the terms $P_{2 n-1}(\cos \theta)$, so

$$
\begin{aligned}
P_{2 n-1}(\cos \theta) \rightarrow P_{2 n-1}(\cos (\pi-\theta)) & =P_{2 n-1}(-\cos \theta), \\
& =-P_{2 n-1}(\cos \theta),
\end{aligned}
$$

due to the odd parity of the Legendre Polynomials with odd coefficients. Thus we have the mirrored potential only changing the signals that accompany the sums in Eqs. (30) and (33). Some interesting points emerge from this study. If we add the two hemispheres (each with the same charge $q$ ), we will have a sphere with uniform density and total charge $2 q$. Evaluating this sum in our solutions only the first term in equation (33) does not cancel (due to signal difference) and we have

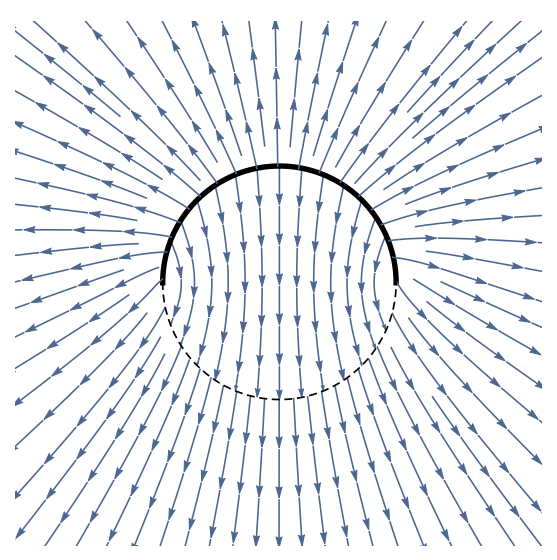

Figure 9: The electric field lines in the $\{x=0\}$ plane for the case 4.4. The continuous black semicircle represents the hemisphere of radius $R$. $\phi^{s p h}(r, \theta)=\phi(r, \theta)+\phi(r, \pi-\theta)= \begin{cases}\frac{(2 q)}{4 \pi \varepsilon_{0} R}, & r \leq R, \\ \frac{2 q)}{4 \pi \varepsilon_{0} r}, & r \geq R .\end{cases}$

As expected, the potential inside the sphere is constant (null electric field) and outside of it corresponds to a Coulombic potential for a point particle with $2 q$-charge. Now, if the inverted sphere has a total charge $-q$ the combination of the two hemisphere results in a "spherical dipole" with potential given by

$$
\begin{aligned}
& \phi_{<}^{\text {dip }}(r, \theta)=\phi_{<}(r, \theta)-\phi_{<}(r, \pi-\theta), \\
& =-\frac{q}{4 \pi \varepsilon_{0} R} \sum_{n=1}^{\infty} \frac{(-1)^{n}}{2^{2 n-1}(2 n-1)} \frac{(2 n) !}{(n !)^{2}}\left(\frac{r}{R}\right)^{2 n-1} \times \\
& P_{2 n-1}(\cos \theta), \quad r \leq R, \\
& \phi_{>}^{d i p}(r, \theta)=\phi_{>}(r, \theta)-\phi_{>}(r, \pi-\theta) \\
& =-\frac{q}{4 \pi \varepsilon_{0} R} \sum_{n=1}^{\infty} \frac{(-1)^{n}}{2^{2 n-1}(2 n-1)} \frac{(2 n) !}{(n !)^{2}}\left(\frac{R}{r}\right)^{2 n} \times \\
& P_{2 n-1}(\cos \theta), \quad r \geq R .
\end{aligned}
$$

The electric field lines are shown in figure 10 . In the interior of the spherical shell, the field is almost homogeneous (mainly around the center). For the outside region, the field lines are closed - an electric dipole.

\subsection{Disc with uniform charge density}

The next two exercises are good examples for understanding the limitations of the method of extension around the axis of symmetry. The first one consists of a disk of radius $R$ and uniform density $\sigma$ (total charge $q=\sigma \pi R^{2}$ ), see figure 11. The calculation of the potential in the z-axis is an illustrative exercise of several basic books [1,2]. The result is

$$
\phi(z)=\frac{q}{4 \pi \varepsilon_{0}} \frac{2|z|}{R^{2}}\left(\sqrt{1+\frac{R^{2}}{z^{2}}}-1\right) .
$$

Because of the parity symmetry $z \rightarrow-z$, only the even Legendre polynomials will contribute in the series

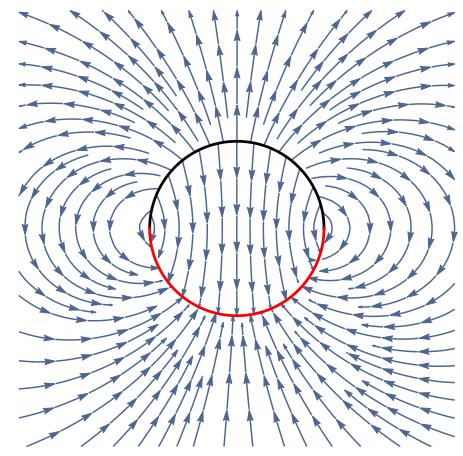

Figure 10: Electric field lines for a spherical shell with positive charge density on one hemisphere (black semicircle) and negative charge density on the other (red semicircle). 


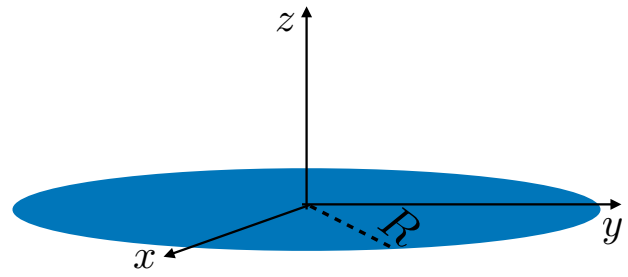

Figure 11: Disc with uniform charge density and radius $R$.

expansion. In all the region $|z|>R$ the scalar potential is the solution of Laplace's Equation; it is thus possible to extract it by the expansion around the axis of symmetry. Expanding equation (36) in powers of $R /|z|$ (see equation 5 (Appendix A) we are left with

$$
\begin{aligned}
& \phi(z)=\frac{q}{4 \pi \varepsilon_{0}} \frac{2|z|}{R^{2}}\left(\sqrt{1+\frac{R^{2}}{z^{2}}}-1\right) \\
& =\frac{q}{4 \pi \varepsilon_{0} R} \sum_{n=1}^{\infty} \frac{(-1)^{n+1}}{2^{2 n-1}(2 n-1)} \frac{(2 n) !}{(n !)^{2}}\left(\frac{R}{|z|}\right)^{2 n-1} .
\end{aligned}
$$

Only the even $B_{l}$ coefficients, i.e. $l=2 n-2(n=1,2, \ldots)$ are nonzero in equation (11). Inserting the Legendre polynomials in the series, the electrostatic potential at all points $r \geq R$ becomes

$$
\begin{aligned}
& \phi_{>}(r, \theta)=\frac{q}{4 \pi \varepsilon_{0} R} \sum_{n=1}^{\infty} \frac{(-1)^{n+1}}{2^{2 n-1}(2 n-1)} \frac{(2 n) !}{(n !)^{2}} \times \\
& \times\left(\frac{R}{r}\right)^{2 n-1} P_{2 n-2}(\cos \theta), r>R .
\end{aligned}
$$

Now is the time to discuss an important point. Naively, one can try to describe the potential in the vicinities of the disc by expanding equation 36 in the region $|z|<R$ and then adding the Legendre polynomials, or by using the inversion method in equation 37 . The two approaches are wrong because on the disk $(0 \leq r \leq R$ and $\theta=\pi / 2)$ the potential is not a solution of Laplace's Equation. The methods of expansion around the axis of symmetry and of inversion can be applied only in cases with azimuthal symmetry and electric charge densities (regions where $\nabla^{2} \phi \neq 0$ ) on surfaces where $r=$ const., $\theta \in\left[\theta_{i}, \theta_{f}\right]\left(0 \leq \theta_{i}\right.$ and $\left.\theta_{f} \leq \pi\right)$. Thus if the potential satisfies Laplace's Equation at the point $z_{0}$ of the $z$-axis, it remains a solution of the same equation at all points of the sphere of radius $\left|z_{0}\right|$. The same holds for the mapping $|z| \rightarrow R^{2} /|z|$ in the inversion method.

For the charged disk, this is not true since the potential is not a solution of the Laplace's Equation at all points $0 \leq r \leq R$ when $\theta=\pi / 2$. Therefore, none of the approaches presented in this article can be used to calculate the potential inside the sphere of radius $R$. In figure 12 the electric field lines are presented for $r>R$. Even in the vicinity of the sphere of radius $R$, the field lines have an approximately radial aspect - the one expected for a point charge.

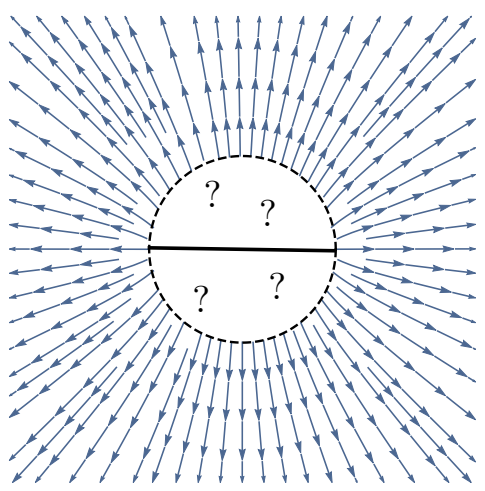

Figure 12: Electric field lines on the plane $\{x=0\}$ for case 4.5 The solid black line represents the disk of radius $R$. It was not possible to obtain the field inside the sphere of radius $R$ (dashed circle).

\subsection{Charged rod}

Our last example is the first solved problem presented in the electric field chapter of almost every basic textbook (e.g., see reference [1]), the rod of length $L$ and uniform charge density $\lambda$ (total charge $q=\lambda L$ ); see figure 13 . Usually, the electrostatic potential is calculated (by simplification) on the plane of symmetry $z=0$, here we need its value on the $z$-axis. Similarly to the case of the disk, it will not be possible to determine the potential at points $0 \leq r \leq L / 2$ and $0 \leq \theta \leq \pi$, since along the line $r=|z|<L / 2$ the potential is not a solution of Laplace's Equation. Our potential is obtained at any point $z>L / 2$ by application of equation $(9)$, then

$\phi(z)=\frac{\lambda}{4 \pi \varepsilon_{0}} \int_{-L / 2}^{L / 2} \frac{d z^{\prime}}{z-z^{\prime}}=-\frac{q}{4 \pi \varepsilon_{0} L} \ln \left(\frac{1-\frac{L}{2 z}}{1+\frac{L}{2 z}}\right)$.

Expanding this in powers of $L / 2 z$, see equation 3 (Appendix A), we have

$$
\begin{aligned}
\phi(z) & =-\frac{q}{4 \pi \varepsilon_{0} L}\left[\ln \left(1-\frac{L}{2 z}\right)-\ln \left(1+\frac{L}{2 z}\right)\right] \\
& =\frac{q}{4 \pi \varepsilon_{0} L} \sum_{n=0}^{\infty} \frac{1}{2^{2 n}(2 n+1)}\left(\frac{L}{z}\right)^{2 n+1} .
\end{aligned}
$$

Comparing with equation 11 we have $A_{l}=0$ and $B_{l} \neq 0$ only for $l=2 n, n \in \mathbb{N}$. The potential at all points $r>L / 2$ is obtained by introducing the Legendre

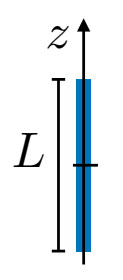

Figure 13: Rod with uniform charge density and length $L$. 


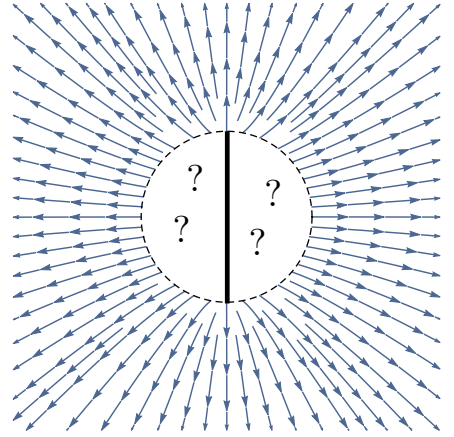

Figure 14: Electric field lines on the plane $\{x=0\}$ for case 4.6 . The solid line represents the bar of length $L$. It was not possible to obtain the field inside the sphere of radius $L / 2$ (dashed circle).

polynomials

$\phi_{>}(r, \theta)=\frac{q}{4 \pi \varepsilon_{0} L} \sum_{n=0}^{\infty} \frac{1}{2^{2 n}(2 n+1)}\left(\frac{L}{r}\right)^{2 n+1} P_{2 n}(\cos \theta)$

$r>L / 2$. In figure 14 we show the electric field generated by this potential. Note that in the vicinity of the equator, $\theta=\pi / 2$, the field lines have a radial behavior (like a point particle), on the other hand they are very different in the point-ends of the $\operatorname{bar}(\theta \approx 0$ and $\theta \approx \pi)$; this is a realization of the edge effects: deformations of the electric field at the ends of finite bodies.

\section{Conclusions}

In this paper, we have constructed in detail two useful methods which can and should be used to solve a large class of electrostatic problems with azimuthal symmetry in a simple way. In cases when the potential can be obtained via direct integration only on the $z$-axis of azimuthal symmetry, the use of the method of extension around the axis of symmetry provides the full solution. The inversion method generates a solution of Laplace's Equation in the range $\left(R / r_{\max }\right) R<r<\left(R / r_{\min }\right) R$ from another solution defined in $r_{\text {min }}<r<r_{\text {max }}$ for some $R \in\left(r_{\min }, r_{\max }\right)$. These two approaches are described in examples in the book Classical Electrodynamics by D. Jackson [6] and briefly in Landau \& Lifshitz Electrodynamics of Continuous Media [7], but both methods are usually not even mentioned in courses of Electromagnetic Theory for undergraduate students. Our main goal here is to call the attention of teachers and students of this discipline to the power and simplicity of these approaches. For this, we presented several illustrative examples. We highlight the case of the ring of charges outside and inside a grounded conducting sphere - these problems are usually studied through complicated methods. It is also important to emphasize the limitations of these approaches. They can be applied in regions when the potential is not only a solution of Laplace's Equation for $r=\left|z_{0}\right|(\theta=0$ or $\pi)$ but is also a solution of the same equation for every $\theta \in[0, \pi]$ (fixed $\left.r=\left|z_{0}\right|\right)$. We show a couple of examples where this does not occur, the disk and the rod, for which the potential was determined only partially. Due to the complexity of the analyzed configurations, all the potentials were described in terms of Legendre polynomials expansions rather than in wellknown functions. However, this is not an impediment to understanding the distribution of the electric field in space. Using the Wolfram Mathematica software, we plotted the electric field lines for all examples discussed in the text. These nice figures bring a visual aspect to the electrostatic phenomena facilitating their qualitative understanding, especially if exposed in an Electromagnetism class. Finally, we hope to motivate the reader to use their creativity to apply these two approaches, perhaps together with the Method of Images, to other exercises of electrostatics.

\section{Supplementary material}

The following online material is acailable for this article: Appendix A - Mathematical supplement

\section{References}

[1] D. Halliday, R. Resnick and K.S. Krane, Física (LTC, São Paulo, 2003), $5^{\mathrm{a}}$ ed., v. 3.

[2] H. Moyses, Curso de Física Básica: Eletromagnetismo (Blucher, São Paulo, 2015), 5 $5^{\mathrm{a}}$ ed., v.3.

[3] R.P. Feynman, R.B. Leighton and M. Sands, The Feynman Lectures on Physics (Basic Books, Nova York, 2001), v. 2 .

[4] D.J. Griffiths, Introduction to Electrodynamics (Cambridge University Press, Cambridge, 2017), 4ª ed.

[5] K.D. Machado, Eletromagnetismo (Toda Palavra Editora, Niterói, 2012), v. 1.

[6] J.D. Jackson, Classical Electrodynamics (Wiley, Nova Jersey, 1998), $3^{\text {a }}$ ed.

[7] L.D. Landau, E.M. Lifshitz and L.P. Pitaevskii, Electrodynamics of Continuous Media: Vol. 8 of Course of Theoretical Physics (Pergamon Press, Oxford, 1984), $2^{\text {a }}$ ed.

[8] R. Weinstock, Am. J. Phys. 44, 392 (1976).

[9] G.B. Arfken, H.J. Weber and F.E. Harris, Mathematical Methods for Physicists: A Comprehensive Guide (Academic Press, Cambridge, 2012), $7^{\mathrm{a}}$ ed. 